\title{
EL CRONOTOPO DE LA NOVELA ESPAÑOLA DE PEREGRINACIÓN: MIGUEL DE CERVANTES
}

« Válame Dios, y con cuánta gana debes de estar esperando ahora, lector ilustre, o quier plebeyo," la segunda parte de estos papeles! Parafraseando a nuestro autor, invocamos al interés de los lectores que conozcan algunas de las reflexiones desarrolladas en un artículo anterior donde tratamos de aplicar la teoría de Mijail Bajtín al estudio de las coordenadas espacio-temporales de la novela de peregrinos.

Para quienes no estén en esas condiciones resumiremos brevemente los lineamientos metodológicos que orientaron nuestra tarea.

El objetivo primordial era, entonces, establecer el grado de resemantización sufrido por el cronotopo de la novela bizantina en la primera actualización del género en España: Los amores de Clareo y Florisea de Alonso Núñez de Reinoso (1552) y El peregrino en su patria de Lope de Vega (1604).

La teoría del cronotopo de Bajtín se mostró como un instrumento crítico capaz de dar cuenta de algunos de los cambios de códigos discursivos provocados por la interacción genérica en esa primera fase del devenir histórico de la novela de amor y aventuras.

Tal como quedó configurada en los dos textos mencionados, los códigos discursivos de la novela española de peregrinos del siglo XVII mostraba los siguientes rasgos:

- es una historia de amor y peregrinación;

- protagonizada por arquetipos cristianos verificados por la superación de pruebas o "trabajos";

- sus episodios son articulados por el cronotopo del camino a través de espacios y tiempos cercanos y definidos;

- las peripecias y anagnórisis son motivadas por la Providencia Divina;

- en ella la peregrinación a Roma pone de relieve la moralidad católica post-tridentina como mensaje ideológico privilegiado.

Sobre esta base, nos proponemos ahora considerar el tratamiento que Miguel de Cervantes hizo del cronotopo de la novela de peregrinación en La española inglesa (1613) y en Los trabajos 
de Persiles y Sigismunda (1617). La escritura cervantina confiere a las novelas de amor y aventuras un sello propio caracterizado por la búsqueda de nuevos límites para la comprensión de sus historias, comprensión que sólo es posible gracias a la estrecha complicidad del narrador con el lector, quien deberá no sólo reconocer lo que se le cuenta sino además evaluarlo desde distintas perspectivas de lectura.

\section{LA ESPAÑOLA INGLESA}

Cervantes sitúa la acción de la novela en un contexto espaciotemporal concreto: el enfrentamiento de España e Inglaterra de fines del siglo XVI y comienzos del XVII. El episodio de la toma de Cádiz en 1596, la presencia de Isabel I, reina de Inglaterra (muerta en 1603), del corsario turco Arnaute Mamí, del conde de Leste (en realidad, Leicester, muerto en 1588), la mención de los trámites comerciales de la reina para enviar el dinero desde Inglaterra a España, así como la orden de la Santa Trinidad; todos estos datos refieren la acción a un periodo histórico concreto aunque impreciso ya que las fechas no coinciden entre sí.

Como era de esperar La española inglesa es la novela ejemplar sobre la que la crítica más ha discutido acerca de la presencia de elementos históricos, en particular porque el texto plantea algunos problemas, por ejemplo, por qué Cervantes construye una atmósfera tan benevolente hacia Inglaterra y los ingleses '. Luego volveremos sobre esta cuestión.

El tratamiento del espacio guarda una relación especialmente significativa con la construcción de la trama, porque la delimitación de dos espacios, Inglaterra y España, permite la incorporación de cronotopos opuestos y complementarios. Por una parte, Isabela se mueve en general por espacios cerrados, la casa, la corte, el convento, y sólo una vez se la presenta en un espacio abierto: la calle, el día en que se dirige al convento a hacer los votos perpetuos. Por otra parte, Ricaredo se desplaza por espacios abiertos, fuertemente cargados de valor emocional: el mar, durante el corso; la ruta, en su peregrinación a Roma; Argel, durante el cautiverio.

Ahora bien, si consideramos el esquema canónico de la novela griega, veremos que Cervantes reemplaza el obstáculo de la separación de los enamorados por oposición de sus padres por una

1 M. Singleton, "The date of La española inglesa,, Hispania, XXX, núm. 3, agosto 1947, pp. 329-335. Allí establece la secuencia 1587-1594-1596, luego desmentida por $\mathbf{R}$. Lapesa que propone como fecha de complosición 1609-1611, periodo de distensión de las relaciones entre ambas naciones. Thomas Hanrahan, "History in the Española inglesas, MLN, 83, n. 2, marzo 1968, pp. 267-271, explica la atmósfera benevolente hacia Inglaterra y los ingleses por haberse compuesto la novela en 1605, cuando los ingleses firmaron la reconciliación con la iglesia de Roma en la ciudad de Valladolid. 
variedad de impedimentos que, progresivamente, van alargando los lapsos cronológicos hasta el reencuentro final. En primer lugar es la reina la que solicita a Ricaredo que parta en corso contra los turcos, luego el celoso conde Arnesto se opondrá a las bodas desafiando a duelo a Ricaredo; más adelante la camarera real, madre del conde, envenenará a Isabela afeándola durante dos meses; finalmente el propio Ricaredo partirá como peregrino hacia Roma «a asegurar su conciencia" prometiendo regresar dos años después. Este alargamiento de los tiempos y la imprecisión histórica es rematada por el narrador quien, antes de cerrar su relato con la moraleja, sitúa la existencia feliz del matrimonio en un presente posible y minucioso en su credibilidad:

(...) en cuya compañia se piensa que aún hoy vive en las casas que alquilaron frontero de Santa Paula, que después las compraron los herederos de un hidalgo burgalés que se llamaba Hernando de Cifuentes ${ }^{2}$.

Hemos soslayado hasta ahora la consideración de un aspecto central en la constitución de los cronotopos en La española inglesa. la novela refiere acontecimientos de la historia contemporánea, y aunque no tome contacto directo con el devenir histórico, como sostiene Bajtín, dichos acontecimientos modifican la configuración de los personajes.

Tomemos por caso el enmascaramiento de los personajes que adquiere la forma del ocultamiento de la verdadera religión, y que puede explicarse como efecto de la inscripción de las circunstancias histórico-políticas de las relaciones entre España e Inglaterra en el texto. Rafael Lapesa plantea la cuestión en los siguientes términos:

Pero lo importante es que tanto la reina como el jefe de la expedición contra Cádiz se muestren humanos con las gentes de distinta religión. Son los dos personajes dirigentes quienes practican la tolerancia. ¿Pensaba acaso Cervantes que esta actuación, no exacta en su referencia al pasado, pudiera corresponder al presente de la política inglesa de su tiempo, o si no, a un futuro deseable y próximo? ${ }^{3}$.

Y responde que es muy posible que Cervantes compartiera la opinión ilusionada de los españoles de que Inglaterra volvería pronto al catolicismo.

Pero volviendo a nuestro punto de partida diremos que esta aproximación a hechos contemporáneos genera una imagen del hombre que, en la escritura de Cervantes, gana complejidad aún en el estatismo. Los personajes de La española inglesa no son

2 M. DE Cervantes, La española inglesa. Novelas Ejemplares. Madrid, Castalia, 1982. La cita, p. 100.

3 R. LAPESA, «En torno a La española inglesa y el Persiles,, De la Edad Media a nuestros dias. Madrid, 197.3, pp. 252-3. 
monolíticos e imperturbables, son complejos porque padecen el conflicto interior ante la realidad que los rodea. Así se explican los temores de Ricaredo cuando sale de corso y se promete no matar cristianos ni turcos, y se explican también las decisiones fundamentales que resuelve Isabela en el umbral del convento. Verdaderos peregrinos fuera de su patria, Isabela y Ricaredo están marcados por la doble identidad denotada por el título de la novela, en el que ambos elementos nominales pueden desempeñar la función de núcleo sustantivo del sintagma. Esta doble identidad surge de cierto grado de iniciativa lingüística del narrador que no fija tipos completamente inmutables sino personajes comprensivos y tolerantes con el otro (sea este turco, católico o anglicano).

En síntesis, los cronotopos de la novela ejemplar de Cervantes configuran una imagen humana matizada por las circunstancias vivenciales que la rodean. Dentro de lo convencional y establecido los personajes de La española inglesa confirman su identidad superando obstáculos pero, como ocurre también en la segunda parte del Quijote, lo hacen aproximándose al devenir histórico, y el narrador expresa dicho acercamiento dándole la forma de una conducta más regida por el imperio del libre albedrío y la sumisión a los dictados de la conciencia, que determinada por los anárquicos designios del azar 4 .

Finalmente si La española inglesa, que tal como afirma la crítica es una prefiguración de Los trabajos de Persiles y Sigismunda, está puesta bajo el imperio de la ejemplaridad y sugiere la imitación de la acción ilustre, destino último de lo verosímil maravilloso, nuestra reflexión sobre las categorías espacio temporales no hace más que completar una definición tentativa del texto. Cervantes ensaya, entre otras cosas, su capacidad narrativa para redefinir el didactismo literario por medio de la combinación de mundos ficcionales distintos (no incompatibles) con unos personajes menos sujetos a lo fortuito, más próximos al devenir, desplazándose por un cronotopo conflictivo: el del enfrentamiento político y religioso de Inglaterra y España. En última instancia, y como es frecuente en la escritura cervantina, todos estos artificios son las piezas en movimiento de un tablero de juegos que el lector recompondrá en la búsqueda de los sentidos últimos de lo dicho: un abanico siempre múltiple de lecturas posibles.

\section{LOS TRABAJOS DE PERSILES Y SIGISMUNDA}

"La plenitud del Persiles como novela fue sacrificada en aras de la más alta intención ideológica», afirma J. B. Avalle-Arce en su

4 Esta reflexión explica las conductas de Isabela y Ricaredo, en tanto que la de la reina de Inglaterra parece sólo justificada por motivos de la coherencia interna del relato $y$, en todo caso, como expresión de deseos de Cervantes, tal como sostiene Lapesa. 
edición del texto, señalando así una observación frecuente en la crítica del Persiles. Y es precisamente esa falta de plenitud, que nosotros interpretamos siguiendo la teoría de Bajtín como un escaso contacto de la novela con el presente histórico en devenir, un efecto inmediato de los cronotopos presentes en la peregrinación de Periandro y Auristela. Luego volveremos sobre el punto de la "alta intención ideológica*.

La presencia de dos bloques narrativos tan definidos como los trabajos septentrionales y los meridionales ha sido largamente elaborada por los críticos ${ }^{5}$. Retomaremos esta cuestión desde la perspectiva de la teoría del cronotopo. El cronotopo del norte que domina los dos primeros libros responde al esquema establecido en la novela griega de aventuras, allí el lazo tempoespacial es puramente técnico y abstracto: no se sabe cuándo ni exactamente dónde Periandro y Auristela se desplazan por una tierra de bárbaros, vagamente presentada como tierras frías y oscuras, generalmente islas, e incluso islas alegóricas como la Isla Maravillosa (II, 15) que nos remite a las ínsulas de Clareo y Florisea ${ }^{6}$. el cronotopo del sur coincide con los libros III y IV e introduce en el relato referencias espacio temporales más precisas y concretas: los peregrinos llegan "diez y siete días" después de navegar desde la isla de las ermitas a Lisboa donde «(....) les pareció que ya habían llegado a la tierra de promisión que tanto deseaban" ?. A partir de ese momento la peregrinación deja de estar sujeta a los avatares e infortunios de la navegación marítima, y sigue un itinerario continental que comprende España, Francia e Italia tras la voluntad expresa de los protagonistas de llegar a Roma. Lejos de la tierra de bárbaros septentrionales, el sur es tierra de pastores y villanos, de templos (Guadalupe, Nuestra Señora de Esperanza) y de mesones, donde Periandro y Auristela son, finalmente, Persiles y Sigismunda.

Esta configuración cronotópica ilumina la reflexión que, por otro camino, hace Riley acerca de los procedimientos narrativos empleados por Cervantes en el Persiles, y el desplazamiento desde el romance (ficción idealista) hacia la novela (ficción realista):

(...) Todos estos indicios de desplazamiento del romance puro, sobre todo en la segunda mitad del libro, señalan al Cervantes novelista que modifica la obra del Cervantes romancista :

5. M. De Cervantes, Los trabajos de Persiles y Sigismunda, Madrid, Castalia, 1970. La cita, p. 27.

- La Insula Maravillosa aparece en el extenso relato que hace Periandro de sus aventuras, con lo cual el narrador se cubre de la posible acusación de faltar contra la verosimilitud del relato, en tanto que delega la responsabilidad de lo dicho a la retórica discursiva del personaje.

' Cervantes, Persiles, ed. cit., p. 277.

- E. C. Riley, "Romance y novela en Cervantes", Cervantes: su obra y su mundo, Actas del I Congreso Internacional sobre Cervantes, Madrid, Edi-6, 1981, 5-13. La cita, p. 13. 
Como muestra de la convivencia de ambas modalidades veremos que, a pocas páginas uno de otro, el narrador echa mano de dos recursos aparentemente contrarios. El primero es la mención de un topónimo presente en las relaciones topográficas de Felipe II, cuando Antonio pregunta:

- ¿Por ventura, señor - $(. .$.$) - este lugar no se llama el Quintanar de la$ Orden, y en él no vive un apellido de unos hidalgos, que se llaman Villaseñores $"$.

En el capítulo siguiente del mismo libro aparece la fórmula quijotesca:

(...) digo que el hermoso escuadrón de los peregrinos, prosiguiendo su viaje, llegó a un lugar, no muy pequeño ni muy grande, de cuyo nombre no me acuerdo, (...) ${ }^{10}$.

Ambos espacios, el histórico y preciso y el olvidado coexisten en el abstracto y difuso tiempo que permite lograr la simultaneidad señalada por Avalle-Arce:

La llegada a la Europa meridional marca el momento de máxima actualidad histórica, pero los personajes $-\mathrm{y}$, por ende, la novela toda- se miran alternativamente, mejor aún, simultáneamente, en la realidad de sus patrias y en la reproducción del mito septentrional ".

De modo que al no precisar fechas, como hizo Lope de Vega en su Peregrino, el narrador maneja con mayor libertad la relación técnica con ambos tiempos ya apuntados.

Una vez más nos planteamos la cuestión de cuál es la imagen del hombre delineada por los cronotopos actuantes en el Persiles. Es inevitable pensar este problema partiendo de lo dicho para $L a$ española inglesa. En ese sentido Periandro y Auristela son distintos a Ricaredo e Isabela, si estos se mostraban como personajes en conflicto interior con sus vivencias, aquellos aparecen sólo ratificados en su identidad por la superación de los obstáculos. Así en términos de elección y destino se definen a ellos mismos por boca de Periandro en I, 16:

Mi hermana y yó vamos llevados del destino y de la elección a la santa ciudad de Roma, y hasta vernos en ella, parece que no tenemos ser alguno, ni libertad para usar nuestro albedrío. Si el cielo nos llevare a pisar la santísima tierra y adorar sus reliquias santas, quedaremos en

- Cervantes, Persiles, ed. cit., p. 334.

I') Ibidem, p. 343. El subrayado es mío.

"J. B. Avalle-ARCE, "Tres vidas del Persiles (Cervantes y la verdad absoluta)", Nuevos deslindes cervantinos. Barcelona, Ariel, 1975, 75-87. La cita, p. 86. El subrayado es mío. 
disposición de disponer de nuestras hasta agora impedidas voluntades (...) ${ }^{12}$.

Más adelante al hablar consigo mismo reflexionando como enamorado dice Periandro en II, 6:

Considera, señora, que el amor nace y se engendra en nuestros pechos, o por elección o por destino: el que por destino, siempre está en su punto; el que por elección, puede crecer o menguar, según pueden menguar o crecer las causas que nos obligan y mueven a querernos ${ }^{13}$.

Por eso debemos volver a lo dicho cuando analizamos la imagen humana en el Peregrino. Los peregrinos cervantinos son estáticos y faltos de iniciativa como lo era el peregrino de Lope. Sólo que no será el azar quien determine la constitución de los cronotopos, sino el cielo, la Providencia Divina, u otro término semejante.

Cervantes en el Persiles sujeta su escritura a los dictados ideológicos de la contrarreforma católica. Ya hemos dicho con anterioridad cuál es uno de los principales condicionamientos ideológicos que más atan al narrador: el peregrino no puede ser un personaje autónomo e incontrolable como el loco manchego. $\mathrm{Si}$ así fuera la peregrinación le daría una visión del mundo más crítica y nihilista, como la que muestra El Licenciado Vidriera. Periandro nunca podría moverse en el cronotopo de don Quijote y Sancho, quedaría invalidado en su excelencia heroica por contacto con el tiempo histórico en devenir donde sólo un personaje que se autoconstruye y elige su destino puede sobrevivir. Pero, al mismo tiempo, únicamente Periandro puede moverse por espacios tan disímiles en un tiempo tan abstracto, porque es un peregrino, extraño en la tierra cuyo único norte y guía es el empeño en llegar a la Jerusalén celeste. En este sentido, la imagen del hombre tal como aparece plasmada en el peregrino Periandro es una imagen sub specie aeternitatis. En tanto que don Quijote es un personaje conformado sub specie humanitatis y como tal, movido por su voluntad y libre albedrío.

Pero no todo es sometimiento y ortodoxia en el Persiles, ya se ha visto cuánto de original y heterodoxo poético muestra la composición de la novela que, por otra parte, no es ni más ni menos que un intento vanguardista de escribir épica en prosa. Y ese intento, tratándose de los dogmas de la fe católica reafirmados por Periandro y Auristela, es la "alta intención ideológica" a la que se refería Avalle-Arce. De allí que puede cuestionarse el concepto de plenitud aplicado a la novela de Cervantes, porque es el primer ensayo previo de hacer en castellano lo que Torcuato Tasso en La Jerusalén libertada, no en vano Cervantes depositaba en ella las mayores expectativas literarias de su vida.

12 Cervantes, Persiles, ed. cit., p. 125.

13 Ibid., p. 185. 


\section{APUNTES PARA UNA CONCLUSIÓN}

Insistiremos en la presentación de dos esquemas que sustentan nuestros apuntes finales aunque no definitivos. Se trata del cuadro 1 que muestra la distribución relativa de los cronotopos en las obras que acabamos de considerar, y del cuadro 2 que proporciona datos relativos a las invariantes genéricas en el uso de los cronotopos y pone en evidencia a aquellos que son exclusivos de un texto en particular.

Cuadro 1: Distribución relativa de los cronotopos en los textos.

La española inglesa

- rapto
- mar
- corte inglesa
- camino
- Roma
- convento
- falsa muerte
- matrimonio

Persiles

$$
\begin{aligned}
& \text { - templo } \\
& \text { - ínsulas } \\
& \text { - mar } \\
& \text { - camino } \\
& \text { - Isla Nevada } \\
& \text { - corte Policarpo } \\
& \text { - Isla de Ermitas } \\
& \text { - Lisboa } \\
& \text { - España } \\
& \text { - templo } \\
& \text { - falsa muerte } \\
& \text { - Francia } \\
& \text { - Italia } \\
& \text { - Roma } \\
& \text { - matrimonio }
\end{aligned}
$$

Cuadro 2: Ocurrencia de los cronotopos en los textos.

\begin{tabular}{lcc} 
& Española & Persiles \\
\cline { 2 - 3 } - camino & $\mathbf{X}$ & $\mathbf{X}$ \\
- convento & $\mathbf{X}$ & $\mathbf{X}$ \\
- corte & $\mathbf{X}$ & $\mathbf{X}$ \\
- falsa muerte & $\mathbf{X}$ & $\mathbf{X}$ \\
- juicio & $\mathbf{X}$ & $\mathbf{X}$ \\
- mar & $\mathbf{X}$ & $\mathbf{X}$ \\
- matrimonio & & $\mathbf{X}$ \\
- naufragio & & $\mathbf{X}$ \\
- posada & $\mathbf{X}$ & $\mathbf{X}$ \\
- rapto & $\mathbf{X}$ & $\mathbf{X}$ \\
- templo & & $\mathbf{X}$ \\
- tormenta & $\mathbf{X}$ & $\mathbf{X}$ \\
- insulas & $\mathbf{X}$ & $\mathbf{X}$ \\
- España & $\mathbf{X}$ & $\mathbf{X}$ \\
- Franciatera & $\mathbf{X}$ & $\mathbf{X}$ \\
- Italia & $\mathbf{X}$ & $\mathbf{X}$ \\
- Portugal & $\mathbf{X}$ & \\
- Roma & &
\end{tabular}


En primer lugar observamos que, mientras La española inglesa delimita espacios geográficamente puntuales en los que Roma es el hito fundamental de la peregrinación, en el Persiles aparecen además los cronotopos abstractos del norte y los concretos del sur constituyendo, de esta forma, un esfuerzo de síntesis en el tratamiento de las coordenadas tempoespaciales.

Una vez más surge la utilización de los cronotopos esenciales del cañamazo bizantino: el camino, la corte, la falsa muerte, el mar, el matrimonio, el rapto, el templo. Sin embargo llama la atención que el cronotopo del juicio, tan relevante en los modelos fundacionales como mecanismo de conformación de la identidad del héroe, no aparezca en los textos cervantinos. Según nuestra lectura Cervantes desecha este recurso y los reemplaza por otro más útil a sus propios fines. Así, el juicio es suplantado por la anagnórisis y el racconto final, permitiéndole al narrador jugar con las expectativas del lector y de los personajes, no sólo en el reconocimiento de la verdadera identidad de los héroes, sino también multiplicando los puntos de vista de la narración y la autorreferencia textual.

En cambio el convento aparece solamente en La española inglesa con el valor de umbral definitivo de la separación de Isabela y Ricaredo, y no casualmente allí se produce el reencuentro de los enamorados y la apoteosis de la belleza irradiada platónicamente por Isabela. Cervantes acude a él sometiéndolo a la economía de elementos narrativos propia de las Novelas Ejemplares y lo pone de relieve cargándolo de valores emotivos: en el convento de Santa Paula está "la monja que tenía la mejor voz», allí Ricaredo es reconocido y cuenta su peregrinación, y también en ese lugar se le pide a Isabela que ponga por escrito su historia. La española inglesa es la única novela que ubica su acción en Inglaterra y resulta obvia la explicación de este hecho.

Por último observamos que en la coordenada temporal, sólo el Persiles utiliza alternativamente el tiempo abstracto y el.concreto. Se podrían exponer múltiples razones de este procedimiento. La más obvia lo relaciona con el planteo norte-sur de las aventuras de Persiles y Sigismunda. Nosotros preferimos acentuar la lectura de este mecanismo como un artificio narrativo esencial en la constitución de la épica en prosa: la libre armonización de la precisión cronológica con la ambigüedad temporal permite al narrador equiparar la peregrinación a Roma de Persiles no sólo con la travesía de Eneas en el texto virgiliano sino también con la peregrinación bíblica del hombre sobre la faz de la tierra en busca de la salvación eterna.

Completamos así un panorama tentativo de la evolución histórica de la novela española de amor y peregrinación: en la primera fase enriqueció los cánones heliodorianos, en especial diversificó los cronotopos y matizó la imagen humana del peregrino; y en la fase siguiente Cervantes elaboró su inserción en los conflictos de 
la historia cercana, y ensayó la forma más ambiciosa de escritura novelesca intentada en España: la épica en prosa. Posteriormente esta especie narrativa acentuó los recursos de sugestión alegórica agotándose a si misma como literatura de entretenimiento.

Indudablemente quedan aún muchos cabos sueltos en el estudio del proceso de conformación de los relatos herederos de Las Etiópicas de Heliodoro en España. Y, como peregrinos somos también los lectores de novelas de peregrinación del siglo XVII, es muy posible que nos reencontremos en alguna otra encrucijada de lecturas.

Emilia I. DefFis de Calvo Universidad de Buenos Aires 